\title{
Study on Correlation of Tidal Forces with Global Great Earthquakes $^{*}$
}

\author{
Youjin Su${ }^{1}$, Hong $\mathrm{Fu}^{1}$, Hui $\mathrm{Hu}^{2}$ \\ ${ }^{1}$ Seismological Bureau of Yunnan Province, Kunming, China \\ ${ }^{2}$ National Astronomical Observatories/Yunnan Observatory, Chinese Academy of Sciences, Kunming, China \\ Email: suyoujin0818@sina.com,huhui@public.km.yn.ac
}

Received February 3, 2012; revised March 6, 2012; accepted April 7, 2012

\begin{abstract}
The correlation between the celestial tidal forces and earthquakes has been a controversial problem, although its research history is very long. This paper analyzes the relation between the tidal forces and all the earthquakes of magnitudes no less than 7.0 which occurred in the entire world from year 1900.0 to 2000.0 by calculating tidal forces and the run tests which yields the runs of earthquakes near the extreme and non-extreme values of the tidal forces. It is shown that the occurrence of an earthquake is relevant to the tidal forces. From the analysis of the relation between the ecliptic longitudes of the lunar ascending node and the seismic activities of the principal seismic belts and regions in the world, it is also shown that the lunar node tide is possibly one of the important astronomical contributing factors of the seismic activities there. The results enrich and support the relevant study of the relation between celestial tidal forces and earthquakes.
\end{abstract}

Keywords: Tidal Force; Earthquake; Run Test; Seismic Belt

\section{Introduction}

The occurrence of a moonquake is closely affected by tidal forces on the moon generated by the Earth and the Sun. This has already been studied and confirmed [1,2]. As for the correlation between the celestial tidal forces and earthquakes, it remains although the research history is very long and many papers have been published it remains controversial problem [3]. The studies of this subject carried out both in China and elsewhere can be roughly divided into two categories:

- The time sequence of certain tidal component is calculated. The statistics of phases corresponding to the earthquake-generating times or the earthquake-generating frequencies are evaluated by different methods [4,5], alternatively, the correlation between the earthquakes and tidal-component is directly found values from the statistics of the tidal periodicity of the earthquake-generating times [6].

- The tidal-force indeed shear stress in the sliding direction of a causative fault is calculated, mainly based on the angle of stress. The relation between the earthquakes and tidal effects is consequently discussed from the result in different aspects $[7,8]$.

Different and even contradicting conclusions have been reached by adopting different methods in these pa-

${ }^{*}$ This work is supported by the tackle key project of the ministry of science and technology of P. R. China (2012BAK19B01). pers. In this paper the method of run test is used. It is different from the methods is previous studies. The relation between the great earthquakes over the world and the celestial tidal forces is thus studied in the perspective of entire time sequence, leading to the conclusion that they do relate to each other. Another difference is that not the earthquakes in a single region but all earthquakes in the world are studied, according to the seismic belts or regions. The phenomenon that earthquakes generally happen with a period of 18.6 years in the main seismic regions of the world suggests that the lunar node tide (i.e. the tidal effect from the Moon at lunar node) should be an important astronomical factor which influences the seismic belts or regions hosting potential new earthquakes.

\section{Celestial Tidal Force Has Close Relation to the Occurrence of an Earthquake}

\subsection{Tidal Force}

The resultant of forces (vector difference) of the lunisolar gravitation exerted on the unit mass of the Earth and the inertial centrifugal force produced from the revolution of the Earth round the common center of mass of the Moon and Earth is called the tide generating force, that is to say, the difference between the gravitation exerted on some part of the attracted celestial object and that exerted on the part with the same mass at the center of the object is 
regarded as the tide generating force (Fang, 1984; Fu 1985; Melchior, 1978). From this the calculation formula of the tide generating force exerted on a particle with a unit mass can be obtained as follows [9]:

$$
\mathrm{F}_{\mathrm{r}}=\frac{\mathrm{KMr}}{\mathrm{D}^{3}}\left(3 \cos ^{2} \mathrm{Z}-1\right)
$$

and

$$
\mathrm{F}_{\mathrm{s}}=\frac{3}{2} \frac{\mathrm{KMr}}{\mathrm{D}^{3}} \sin 2 \mathrm{Z}
$$

here $\mathrm{F}_{\mathrm{r}}$ and $\mathrm{F}_{\mathrm{s}}$ are respectively the vertical and horizontal components of the tide generating force, with $F_{r}$ being taken as negative when pointing to the geocentre and $F_{s}$ being positive when pointing to the celestial object, $\mathrm{K}$ is the gravitational constant, $\mathrm{r}$ is the earth's radius, and $\mathrm{M}$ is the mass of the celestial object. $\mathrm{D}$ is the distance from the object to the geocentre. $Z$ is the zenith distance of the celestial object. From the above-mentioned formula it can be calculated that though the solar mass is 2.7 million as much as the lunar mass, the distance between the Moon and Earth is only 1/390 of the distance between the Sun and Earth, and therefore the tide raising force of the Moon is 2.25 times as much as that of the Sun, with both belonging to the same order of magnitude while the tidal force on a point of the earth's surface exerted by each of the other planets is several orders of magnitude less than that exerted by the Moon, and therefore the tidal forces of the other planets can be neglected.

\subsection{Run Test}

One of the most strong points of the run test is that the distribution of the stochastic variable need not be taken into account and it is a nonparametric method. A sequence is considered which consists of $n$ observed values of the stochastic variable $\mathrm{x}$. The variable $\mathrm{x}$ is divided into two kinds which repel each other, and can simply be expressed with the positive sign $(+)$ and negative sign (-). The simplest example is the sequence, which is obtained by throwing a coin, with each observed value being either positive $(+)$ or negative $(-)$. The second example, assuming that a sequential measured value is $\mathrm{x}_{\mathrm{i}}$ (where $\mathrm{i}$ $=1,2,3, \cdots, \mathrm{n})$ and their mean value is $\mathrm{x}_{0}$, and then it is $(+)$ when $x_{i} \geq x_{0}$ and it is $(-)$ when $x_{i}<x_{0}$. The third example, a double sequence consists of two serial observations $\mathrm{x}_{\mathrm{i}}$ and $\mathrm{y}_{\mathrm{i}}$ (where $\left.\mathrm{i}=1,2,3, \cdots, \mathrm{n}\right)$, where each pair of the observations are $(+)$ when $x_{i} \geq y_{i}$ or $(-)$ when $x_{i}<$ $y_{i}$. In any case the observed sequence obtained by the positive sign $(+)$ and negative sign $(-)$ can be written as:

$$
\begin{array}{ccccccccccc}
++- & ++- & +++ & + & --+ & -- & + & --- \\
1 & 2 & 3 & 4 & 5 & 67 & 8 & 9 & 10 & 11 & 12
\end{array}
$$

A run is defined as a segment of the sequence to include the same category of outcomes as many as possible. There are $\mathrm{N}=20$ of runs observations in the above illus- tration, with the number of runs being $r=12$. The number of an observational sequence shows whether the outcomes of the stochastic variable are independent or correlated. The run test can be directly used for analyzing data and a test for the existence of systematic pattern of a single observational sequence may be given as follows.

Let the assumption be that there is no clear pattern in a sequence which consists of $\mathrm{N}$ independent observations of the same stochastic variable. Under the assumption that the observations with + are equal to those with - , the sampling distribution of the number of runs in the sequence is determined by the run-distribution theory. From the comparison of the observed number of runs to the opened interval $\left(\mathrm{r}_{\mathrm{n}}, 1-\alpha / 2, \mathrm{r}_{\mathrm{n}}, \alpha / 2\right)$, the assumption can be tested under any required level of significance $\alpha$, where $n=N / 2$. If the number of runs lies inside this opened interval the assumption is accepted within the confidence level. Otherwise, the assumption is rejected as not having sufficiently confident evidence [10].

\subsection{Test of the Influences on Great Earthquakes from the Celestial Tidal Forces}

Engdahl and Villasenor [11] collected the statistics of seismic records of all countries in the world and found, as a result, that the records of earthquakes of $\mathrm{M} \geq 7.0$ have been complete and reliable since 1900. In order to determine whether the occurrence of an earthquake is correlated with the celestial tide generating force or not, and to let the conclusions obtained from analysis be reliable and reveal the true characteristics of the matter, all the earthquakes events analyzed in this article are selected from the "Global seismicity: 1900-1999" with Engdahl and Villasenor (2002). There occurred 1553 earthquakes of $M \geq 7.0$ in the whole world in the time interval from 1900.0 to 2000.0 , and there were 67 earthquakes of $M \geq 8.0$ during that same interval. The analysis in the present paper is carried out for all the earthquakes with $\mathrm{M} \geq 7.0$.

It is common knowledge that the energy E released by an earthquake and its magnitude $\mathrm{M}$ have the following relation [12]:

$$
\mathrm{E}=10^{11.8+1.5 \mathrm{M}}
$$

here $\mathrm{M}$ correspond the magnitude determined by surface wave. Unit of E is erg. Form which it is not difficult to know that if earthquake is one order greater than another in magnitude the energy released by the former will be 30 times greater than the latter. Thus as far as the energy released by earthquakes is concerned, it is mainly concentrated on great earthquakes. The destruction caused by earthquakes mainly also comes from the great earthquakes.

It is considered that all over the word the earthquakes of $\mathrm{M} \geq 8.0$ are too few while those of $\mathrm{M}<7.0$ are too 
many. In addition, there are some earthquakes of $\mathrm{M}<7.0$ caused by artificially created factors (such as the nuclear explosion, construction of large dams and reservoirs, etc.), and the reliability of the catalogue of the earthquakes of $\mathrm{M}<7.0$ is not good as that of $\mathrm{M} \geq 7.0$. Based on these reasons all the earthquakes of $M \geq 7.0$ are selected by us as the object for analyses. As for analyses of every year we take the frequencies of the earthquakes of $M \geq 7.0$ in that year and do not compute their energies.

Based on the time of occurrence of an earthquake and the longitude and latitude of the epicenter, the celestial tidal force exerted on the epicenter when the earthquake occurs is calculated and the run tests are respectively given to three sequences of the tidal force (i.e., the resultant, vertical component and horizontal component of the tide generating force), or the runs at which the earthquake occurs near the extreme and non-extreme values are calculated. It is found from the results that for the resultant, vertical component and horizontal component, the runs at which the earthquake occurs near the extreme and non-extreme values are respectively 1030, 1046 and 510. The null hypothesis is taken as that the occurrence of the earthquake has no relation to the celestial tidal force, then for the level of significance $\alpha=0.05$, the acceptance region of the assumption $\mathrm{n}=\mathrm{N} / 2=776$ should lie in the opened interval $(656,906)$. Evidently, all the runs of the above mentioned three sequences lie outside this opened interval, and therefore the null hypothesis is rejected. In other words, the occurrence of an earthquake and the tidal force have obvious potential tendency, i.e. the occurrence of an earthquake has some relation to the celestial tidal force.

\section{Seismic Activities of Principal Seimic Belts in the Whole World and Lunar Node Tide}

\subsection{Existence of Period of 18.6 Years in Seismic Activities in Principal Seismic Belts in the World}

As already pointed out, among various celestial tidal forces the lunar-origin tidal force is the greatest, and the next important is the solar-origin tide fore. Therefore the tidal force of the lunar origin becomes the prior subject in our study. During the studies of the characteristics of seismic activities, many researchers have found that the earthquakes in a certain region or belt have periodicity in activities $[13,14]$. There were 5 active periods of strong earthquakes and 5 calm periods in China in the 20th century [15] with the period being 18.6 years [16]. There may be similar periodicity of earthquakes over the entire world as well. If the earthquakes of $M \geq 7.0$ in the entire in the 20th century are collectively analyzed, there is no evidence for such periodicity. However, the period of 18.6 years apparently exists for the earthquakes in a limited region associated with boundaries between tectonic plates. The phases of the cycles of earthquake frequentcies in different seismic belts or regions are different. This causes the period of 18.6 years not seen in the data covering the entire world. The differences in phases are understandable. Because the occurrences of earthquakes are related to certain geological structures, only in the areas with the same or similar seismotectonics, their seismic activities can have the same or similar laws. Therefore, the earthquakes in different regions need to be studied separately, otherwise little pattern of the seismic activities can be found. It is the very reason why the regularity of the seismic activities has not been revealed from the analyses of the combined data of all earthquakes of $\mathrm{M} \geq 7.0$ over the world.

As pointed out by Chen Yong, regularity and randomness coexist in seismic activities, of which above $90 \%$ are distributed near the boundaries between tectonic plates [17]. The theory of tectonic plates holds that the crust of the Earth consists of some rigid blocks or plates, the interior of a plate is relatively stable, many phenomena, such as submerging, colliding and shearing, occur at the boundaries between the plates and the mutual movements between the plates cause the formations and occurrences of earthquakes. A plate drifts under the joined influence of the centripetal force from the Earth rotation and the mantle plume heat convection. The Celestial tidal forces periodically trigger the release of the stress energy accumulated along the boundaries between plates, in the forms of the seismic energy and volcanic energy [18].

Since the major earthquakes are mainly concentrated near the boundaries between the tectonic plates [19,20], we divided the seismic belts of the major earthquakes over the world along the plate boundaries into 11 regions (Figure 1). We find for separate analysis that the seismic activities of each region have a period of 18.6 years. There are 1416 earthquakes of $M \geq 7.0$ in the 11 regions in total making up $91.2 \%$ of all the earthquakes of $\mathrm{M} \geq$ 7.0 of the world. The remaining 137 earthquakes of $M \geq$ 7.0 did not appear to be in the 11 regions.

\subsection{Lunar Node Tide Is an Important Earthquake-Pregnant Astronomical Factor in the Principal Seismic Belts and Regions in the World}

The period of 18.6 years equals to the period of the Earth main nutation term, and also of the motion of the lunar node. The Moon revolves around the Earth in its orbit, while the lunar orbit shifts under the action of the solar gravitation. Therefore, the node of the lunar orbit on the ecliptic (i.e., the plane of the Earth's orbit) moves westward, causing the ecliptic longitude of the lunar ascending node to steadily decrease. The ecliptic longitude of the lunar ascending node is expressed by the equation [21]: 


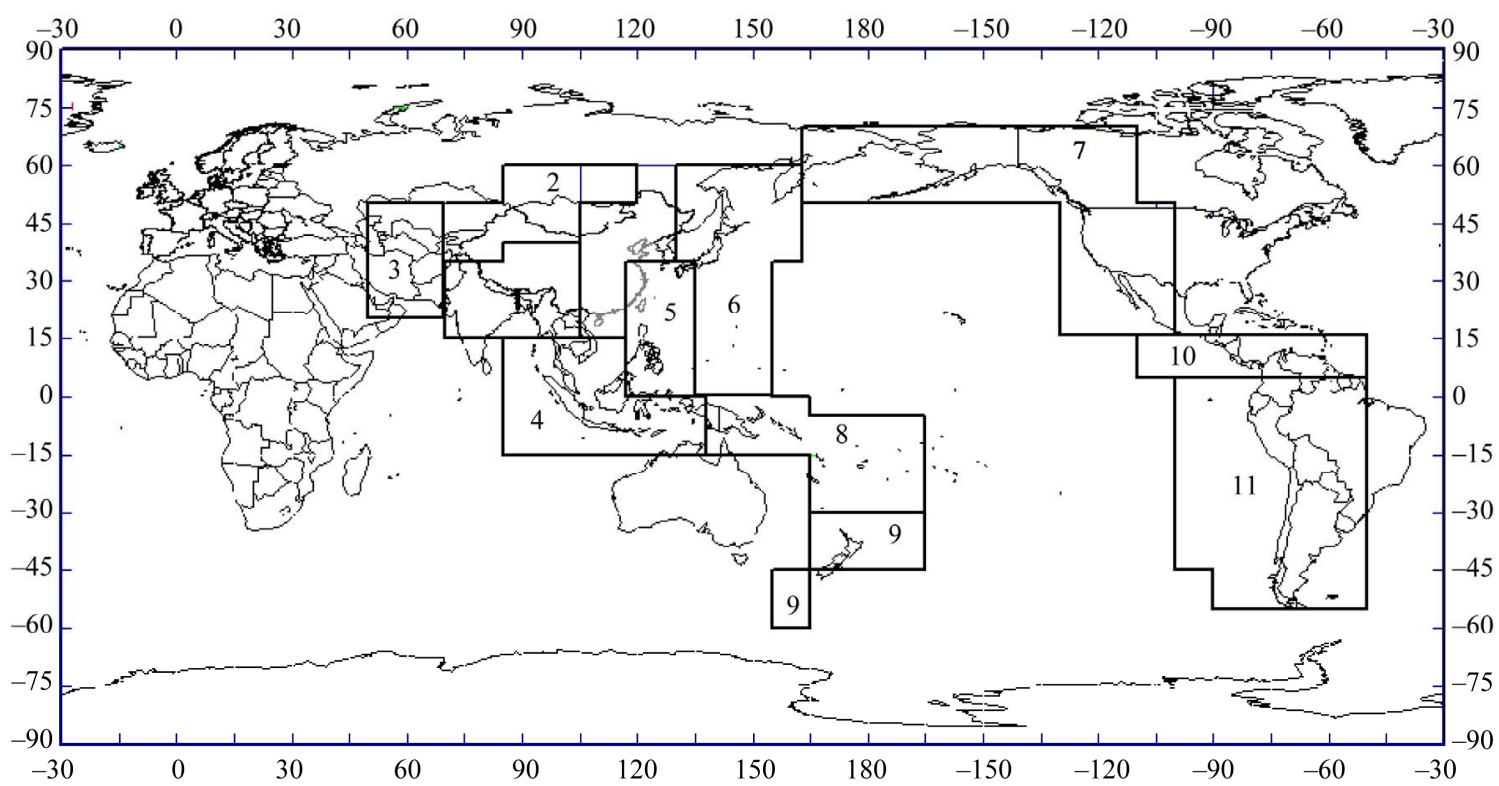

Figure 1. The studied region of $\mathbf{1 8 . 6}$ years recurrence of the great earthquakes in the world.

$$
\begin{aligned}
\Omega= & -125^{\circ} 02^{\prime} 40.280^{\prime \prime}-1934^{\circ} 08^{\prime} 10^{\prime \prime} .539 \mathrm{~T} \\
& +7^{\prime \prime} .455 \mathrm{~T}^{2}+0^{\prime \prime} .008 \mathrm{~T}^{3}
\end{aligned}
$$

where $\mathrm{T}$ is the number of Julian centuries elapsed from J2000.0. This equation can be used to calculate the longitude of the lunar ascending node for the moment of any earthquake. The distribution of the ecliptic longitudes of the lunar ascending node at the time of earthquakes in the region under study can thus be obtained. This allows us to find potential concentration patterns of the ecliptic longitude of the lunar ascending node at the time of occurrence of the earthquakes in each studied region, i.e. including the periods of the seismic activities there. By taking the uniform distribution as the null hypothesis we carry out the $\chi^{2}$ test for each region. A sampling point is taken in the active period or in the calm period, and the degree of freedom evidently equals to one. Under degree of freedom is equal to 1 , if $\chi^{2}>3.841$, the null hypothesis can be rejected with the 95 percent this confidence level, and the periodicity of earthquakes to concentrate within the mentioned active period is highly likely. The test of results show that the values of $\chi^{2}$ are all greater than 3.841 as listed in Table 1. Therefore, the seismic activities in each region studied have significantly shown a period of 18.6 years. The interval of strong seismic activities $\mathrm{N}$ is associated with an opened interval of the ecliptic longitude of the lunar ascending node in the Table 1.

The tidal forces of solar and lunar origins not only cause the sea tides on the Earth surface but also deform the elastic Earth mantle, consequently redistribute the matter and change the gravitational field of the Earth $[22,23]$. The tidal force of the lunar origin is 2.25 times as much as that of the solar origin. During the steady westward motion of the lunar ascending node, the obliquity between the lunar orbit and to the ecliptic varies in the range $4^{\circ} 57^{\prime}$ to $5^{\circ} 19^{\prime}$, thereby causing the obliquity between the lunar orbit and the Earth equator to vary, in the range $18.4^{\circ}$ to $28.8^{\circ}$, with a period of 18.6 years. The variation in the obliquity between the lunar orbit and the Earth equator translates into the variation in the range of the declination of the lunar motion in a tropical month and ultimately the latitude range of strong tidal effects of lunar origin on the Earth surface, thereby controlling the range of motion of the Moon in the equatorial zone and evidently controlling the latitude zone of the tidal force on the Earth's surface affected by the Moon. Therefore, the influence of the inclination of the Moon's path with the equator results in the tidal force exerted by the Moon on the Earth's surface.

It is well known that the pattern of seasons in the southern hemisphere of the Earth is opposite to that of the northern hemisphere due to the difference between the amounts of solar radiation received (which in turn depend upon the incident angles of the sunlight beams), e.g., when there is a hot summer in the southern hemisphere, there is a severe winter in the northern hemisphere (Pen \& Lu, 1983). The most fundamental reason for this phenomenon is that there exists the obliquity of the ecliptic from the equatorial plane of the Earth. Simi larly the obliquity of the Lunar orbit from the terrestrial equatorial plane is probably the astronomical factor causing the latitude dependence of the seismic activities.

\section{Discussion}

1) A new method, which is to study earthquakes in 
Table 1. Statistic results of seismic recurrence of $\mathbf{1 8 . 6}$ years of the principal seismic regions in the whole world.

\begin{tabular}{lcccc}
\hline \multicolumn{1}{c}{ No. Studies region } & Total sample & \multicolumn{3}{c}{ Seismic active interval } \\
\cline { 3 - 5 } & & Sample & N & $\left({ }^{\circ}\right)$ \\
\hline 1) Himalayas and its region & 48 & 45 & $(260180)$ & 9.000 \\
2) Mt.Tianshan and Baikal & 45 & 37 & $(0270)$ & 4.900 \\
3) Eastern Alps plate & 19 & 15 & $(0200)$ & 5.221 \\
4) Nalay Penisaula-Sund Islands & 110 & 86 & $(70330)$ & 3.958 \\
5) Weslern Philipping sea plate & 187 & 162 & $(15080)$ & 8.480 \\
6) Philippine sea-Northeast Japan & 236 & 168 & $(13010)$ & 16.041 \\
7) Aleut-North Mexico & 132 & 73 & $(0250)$ & 6.303 \\
8) New Guinea-Cumtuok & 371 & 355 & $(350330)$ & 7.851 \\
9) South Cumtuok & 30 & 26 & $(60330)$ & 8.244 \\
10) Central America Caribbean & 71 & 59 & $(350230)$ & 4.187 \\
11) West of southern America & 167 & 126 & & 9.674 \\
Total & 1416 & 1152 & & \\
\hline
\end{tabular}

Total sample is the time of occurrence of an earthquake in the studies region from 1900.0 to 2000.0 ; $\mathrm{N}$ is an opened interval of strong seismic activities with the ecliptic longitude of the lunar ascending node; Sample is the time of occurrence of an earthquake in the opened interval N.

separate regions and employs run tests, is adopted in this paper. If the earthquakes of the entire world are considered collectively, there is no evidence of the period of 18.6 years. The situation changes little if the earthquakes in apparently ill-defined regions are studied. The key to the question is to divide earthquake regions reasonably. Through the run tests of the occurrences of earthquakes near the extreme values and non-extreme values of the celestial tidal forces we reach the conclusion that the occurrences of earthquakes are related to the celestial tidal forces. We thus emphasize the importance of adopting proper regions of seismic activities and using the run tests in probing the influences of tidal forces on earthquakes.

2) The contribution of astronomical factors on the earth is a global effect. However, at different time one of the astronomical factors plays a role in different region on the earth. Response regions are confined to the area where potential movement in the atmosphere and crust is highly unstable. Therefore natural calamities induced by astronomical factors are regional effects [24].

3) The variation of the obliquity of the lunar orbit causes variation in the tidal force of the lunar origin. The amplitude of the tidal force of the lunar node is smaller than that of the tidal force at the semidiurnal diurnal, or leading to semi-monthly tides (Gao, 1997). Studies have already verified that the tidal forces of the semidiurnal, diurnal, and semi-monthly tides play triggering roles in the earthquakes in some regions [25-30]. According to the theory of dissipative structures, when the earthquakeformation and energy accumulation have already been in an extreme unstable state, which is far away from the equilibrium, any small fluctuation can be enlarged to cause the giant fluctuation of the geological system, thereby inducing an earthquake [31]. The so-called trigger means that an earthquake may be triggered at any moment when the earthquake-pregnency has arrived at the moment of occurrence of the earthquake. So that the lunar node tide may not play a trigger role in the occurrence of an earthquake or may and probably it participates in the earthquake-pregnant activities, or the longterm action of the lunar node tide joins the earthquakepregnant process in the seismic belt and the accumulative effect of its action is added to the accumulation of the regional energy and to deviate the area from slowly stable $[32,33]$. The details of such modulation mechanism need to be studied in future.

4) The period of 18.6 years in the seismic activities is only statistical. From Table 1 it can be clearly seen that except for the phase differences, the patterns of cycles of different regions are also different: some have longer active times and some have considerably short active times. An earthquake is an extremely complicated process, as it is affected by many factors internal and external to the Earth's (the internal factors are more important, since the external factors affect through the internal factors), and therefore, earthquakes cannot have exact periodically.

\section{Acknowledgements}

We heartily thank for the referee's valuable comments. In response to our request, Professor E. R. Engdahl of the University of Colorado at Boulder (USA) sent us twice their paper and computer readable catalogue of global earthquakes, which is adopted in this paper. He also gave 
us tremendous help in the process of this work. Dr. Chen I-wan, British (ancestor Chinese), Advisor of Committee of Natural Hazard Prediction of China Geophysics Society gave us valuable discussion and help. We express our heartfelt thanks to these colleagues.

\section{REFERENCES}

[1] N. R. Goutly, "Tidal Triggering of Deep Moonquakes," Physics of the Earth and Planetary Interiors, Vol. 9, No. 1, 1979, pp. 52-58.

[2] M. N. Toksöz, "Lunar and Planetary Seismology," Reviews of Geophysics and Space Physics, Vol. 13, No. 3, 1975, pp. 306-311. doi:10.1029/RG013i003p00306

[3] X. P. Wu, et al., "Statistical Analysis of Tidal Stress Effect on Seismic Faults," Chinese Journal of Geophysics, Vol. 42, 1999, pp. 65-74.

[4] S. Shlien, "Earthquake-Tide Correlation," Geophysical Journal. Royal Astronomical Society, Vol. 28, No. 1, 1972, pp. 27-34. doi:10.1111/j.1365-246X.1972.tb06108.x

[5] H. Tsuruoda, M. ohtake and H. Sato, "Statistical Test of the Tidal Triggering of Earthquakes: Contribution of the Ocean Tide Loading Effect," Geophysical Journal International, Vol. 122, No. 1, 1995, pp. 183-194. doi:10.1111/j.1365-246X.1995.tb03546.x

[6] P. R. Du, "18.6 Years Seismic Cycle and the Preliminary Exploration for Its Cause," Chinese Journal of Geophysics, Vol. 37, No. 3, 1994, pp. 362-369.

[7] T. H. Heatön, "Tidal Triggering of Earthquakes," Bulletin of the Seismological Society of America, Vol. 72, No. 6, 1982, pp. 2181-2200.

[8] M. Souriau, A. Souriau and J. Gagnepain, "Modeling and Detecting Interactions between Earth Tides and Earthquakes with Application to an Aftershock Sequence in the Pyrenees," Bulletin of the Seismological Society of America, Vol. 72, No. 1, 1982, pp. 165-180.

[9] G. B. Peng and W. Lu, "The 4th Sort of Natural Factors of the Climate," Science Press, Beijing, 1983.

[10] J. S. Bendat and A. G. Piersol, "Random Data: Analysis and Measurement Procedures," 1971.

[11] E. R. Engdahl and A. Villasenor, "Global Seismicity: 1900-1990,” In: H. K. Lee, H. Kanamori, P. C. Jennings, et al., Eds., International Handbook of Earth- quake and Engineering Seismology, Part A, Academic Press, Amsterdam, 2002. doi:10.1016/S0074-6142(02)80244-3

[12] Z. L. Shi, “The World's Earthquake Catalogue," Seismolog Press, Beijing, 1981.

[13] S. Z. Hong, "Application of the Optimum Section to Seismic Instalments," Northewest Seismology Journal, Vol. 6, No. 1, 1984, pp. 49-57.

[14] G. M. Zhang and L. M. Gen, "Computer Simulation Study of the Recurrent Activities of the Strong Earthquakes in Chinese Mainland," Earthquake Research in
China, Vol. 9, No. 1, 1993, pp. 20-22.

[15] B. X. Gao, "Principle of Astro-Geodynamics," Science Press, Beijing, 1997, pp. 31-50.

[16] H. Hu and X. M. Li, "Research on Correlation of Positions of Celestial Objects with Earthquakes," Natural Hazards, Vol. 23, No. 2-3, 2003, pp. 339-348.

[17] Y. Chen and P. J. Shi, "Natural Disaster," Beijing Normal University Press, Beijing, 1996, pp. 70-71.

[18] X. C. Jin, "Theory of Structure of the Plates," Shanghai Science and Technology Press, Shanghai, 1981, p. 310.

[19] GINSB, "Seismic-Tectonic Map of Europ-Asia," Map Press, Beijing, 1981.

[20] G. W. Moore, "Plate-Tectonic Map of the Circum-Pacific Region," The America Association of Petroleum Geologists, 1982.

[21] D. D. McCarthy Ed., "IERS Standards," IERS Technical Note 3, Observatoire de Paris, 1989, pp. 1-77.

[22] P. Melchior, "The Tides of the Planet Earth," Pergamon Press, Oxford, 1978.

[23] J. Fang, "Solid Earth Tides," Science Press, Beijing, 1984.

[24] Z. J. Guo, et al., "Earth-Atmosphere Coupling and Natural Disasters Forecast," Seismological Press, Beijing, 1996.

[25] F. W. Klein, "Earthquakes Warms and the Semidiurnal Solid Tide," Geophysical Journal. Royal Astronomical Society, Vol. 45, No. 2, 1979, pp. 245-295.

[26] D. Young and W. Zurn, "Tidal Triggering of Earthquakes in the Swabian Jura?" Journal of Geophysics, Vol. 45, No. 1, 1976, pp. 171-182.

[27] A. Palumbo, "Lunar and Solar Tidal Components in the Occurrence of Earthquake in Italy," Geophysical Journal. Royal Astronomical Society, Vol. 84, No. 1, 1986, pp. 93-99. doi:10.1111/j.1365-246X.1986.tb04346.x

[28] P. A. Rydelek, I. S. Sacks and R. Scarpa, "On Tidal Triggering of Earthquakes at Campi Flegrei, Italy," Geophysical Journal International, Vol. 109, No. 1, 1992, pp. 125-147.

[29] X. M. Gao, et al., "Triggering of Earthquakes by the Tidal Stress Tensor," Acta Seismologica Sinica, Vol. 3, No. 3, 1981, pp. 264-275.

[30] J. Zhao, Y. B. Han and Z. A. Li, "Variation in the Solar and Lunar Tide Raising Forces and Earthquakes in Taiwan," Journal of Natural Disasters, Vol. 10, No. 4, 2001, pp. 137-141.

[31] L. D. Chen, "Cerebration of the Scientific Guide Lines, Methodology and Difficulties in Earthquake Prediction," Seismological Research, Vol. 15, No. 2, 1996, pp. 67-74.

[32] P. R. Du and D. Y. Xu, "Astroseismologic Introduction," Seismological Press, Beijing, 1979.

[33] J. Q. Luan, "Motion of Celestial Object and Long-Term Prediction of Weather and Earthquakes," Beijing Normal University Press, Beijing, 1988. 